\title{
Rethinking the autonomic nervous system: genetics and cell fate
}

\author{
Frances Lefcort ${ }^{1}$ (])
}

Received: 14 December 2017 / Accepted: 15 December 2017 / Published online: 27 December 2017

c) Springer-Verlag GmbH Germany, part of Springer Nature 2017

Gaskell and Langley's classical studies from the nineteenth and twentieth century described the autonomic nervous system (ANS) outflow as being divided into three neuraxial regions: a thoraco-lumbar sympathetic circuit bracketed by cranial, brainstem parasympathetic and sacral parasympathetic circuits. These and subsequent anatomical, physiological and pharmacological studies formed the foundational basis for our organizational understanding of the ANS with preganglionic neurons in the brainstem and sacral cord innervating parasympathetic postganglionic neurons that were in close proximity to their targets, while thoracolumbar preganglionic neurons innervated paravertebral and pre-vertebral sympathetic ganglia that tended to be further away from their target organs. Jean-Francois Brunet and colleagues challenged this classification schema in 2016 [1] with the surprising finding that neurons in the "sacral parasympathetic" outflow circuit express 15 different phenotypic and ontogenetic features of pre- and postganglionic sympathetic neurons, rather than of pre- and postganglionic parasympathetic neurons. These genetic and developmental data argue against a distinction in phenotype between thoracic and sacral spinal preganglionic neurons, and rather indicate that the entire spinal ANS outflow-from thoracic to sacral-is sympathetic, while only the cranial brainstem output is parasympathetic.

With the revolution in molecular genetics, identification of potent patterning transcription factors, the ease of in vivo molecular gain- and loss-of-function experimental manipulations and advances in microscopy, the developmental lineages of both brainstem and spinal motor neurons have been mapped over the past 30 years. These studies have identified distinct genetic codes that specify the stereotyped fate of cells in the brainstem and spinal cord. Importantly, these studies reveal that the genetic lineage

Frances Lefcort

lefcort@montana.edu

1 Department of Cell Biology and Neuroscience, Montana State University, Bozeman, MT 59717, USA of brainstem preganglionic neurons is distinct from that of spinal preganglionic neurons - thoracic to sacral-and that the latter depend on the transcription factor Olig2 for their differentiation, whereas, all brainstem preganglionic neurons depend on the transcription factor Phox $2 b$. These differences are not merely descriptive, but in fact, based on functional genetic manipulations in vivo. Moreover, recent studies have also re-evaluated the origin of parasympathetic ganglia and have found that their development is distinct from that of sympathetic ganglia [2, 3]. All postganglionic parasympathetic neurons arise from a genotypically distinct subpopulation of (neural crest-derived) Schwann cell precursors that migrate along the axons of parasympathetic preganglionic neurons to the location of the future parasympathetic ganglion. This is in contrast to how sympathetic ganglia arise, which is directly from migrating neural crest cells at the target site. Pelvic ganglia apparently develop like all other sympathetic ganglia: the spinal preganglionic axons are not associated with the specific subpopulation of Schwann cell precursor cells required to give rise to postganglionic parasympathetic neurons, and the pelvic ganglion forms normally if the preganglionic nerve is significantly reduced in size. In summary then, the developmental genetics underlying the sacral autonomic outflow, both its preganglionic and postganglionic components, meet the criteria of being sympathetic rather than parasympathetic.

Not surprisingly, this paradigm shift garnered the field's attention $[4,5]$. The major refutations involve the type of functional output, location of preganglionic inputs and postganglionic targets, and finally the neuropharmacology and connectivity of the pelvic ganglion. In a new comprehensive article published in this issue of Clinical Autonomic Review [6], Espinosa-Medina et al. have room to expand on their reclassification rationale and argue convincingly that the weight of their ontogenetic data refute the anatomical, physiological and pharmacological evidence underlying the classical model. A major focus of the controversy stems from the complicated biology and neurochemistry of the pelvic ganglion: superficially, its neuropharmacology is neither classically sympathetic nor parasympathetic as it 
contains both adrenergic and cholinergic neurons. Although it's well known that subsets of other (bona fide) sympathetic neurons are cholinergic, for example, those that innervate the sweat glands, periosteum and some blood vessels, the pelvic ganglion is more heterogeneous than a standard autonomic ganglion: for example, its spinal preganglionic input is segregated with the sacral spinal cord projecting primarily to cholinergic pelvic ganglion neurons while the lumbar cord projects primarily to noradrenergic pelvic neurons-an organization consistent with the classical paradigm of dual, integrated suprasegmental control of pelvic target organs.

Given the agreed upon complex circuitry and unconventional neurotransmitter expression of pelvic ganglion neurons (which apparently is also shared to some degree with the bona fide sympathetic inferior mesentery ganglion), Espinosa-Medina and colleagues also thoroughly re-evaluate the physiological studies on the lumbosacral antagonistic control of the bladder and erection, including the evidence for vasoconstrictor and vasodilator pelvic ganglion neurons, and find the data either "discordant" (former) or "evaporated" or at best, "questionable" (latter). Having discarded dual antagonistic lumbosacral control of these targets, the authors address the remaining issue of whether collectively the data indicate that the sacral output is sympathetic or parasympathetic. The authors argue that the strength of their ontogenetic data outweigh the controversial physiological, anatomical and pharmacological data in favor of it being sympathetic. In fact, the authors argue their reinterpretation of the classical schema better explains the confounding and often contradictory historical experimental physiological and pharmacological data.

As with all novel and provocative findings, this work raises many interesting questions for future study that will hopefully reconcile and integrate the complex sacral outflow physiology with genetic dissection of the sacral neural circuits. Comprehensive comparative single-cell transcriptional profiling and advanced connectomics analyses on adult preand postganglionic neurons should reveal the repertoire of molecular and functional diversity exhibited by neurons not only in the unusual pelvic region of the ANS, but throughout the neuraxis.

Acknowledgements This work was supported by NIH R01 NS086796.

\section{References}

1. Espinosa-Medina I, Saha O, Boismoreau F et al (2016) The sacral autonomic outflow is sympathetic. Science 354:893-897

2. Dyachuk V, Furlan A, Shahidi MK, Giovenco M, Kaukua N, Konstantinidou C, Pachnis V, Memic F, Marklund U, Müller T, Birchmeier C, Fried K, Ernfors P, Adameyko I (2014) Neurodevelopment. Parasympathetic neurons originate from nerve-associated peripheral glial progenitors. Science 345(6192):82-87

3. Espinosa-Medina I, Outin E, Picard CA, Chettouh Z, Dymecki S, Consalez GG, Coppola E, Brunet JF (2014) Neurodevelopment. Parasympathetic ganglia derive from Schwann cell precursors. Science 345(6192):87-90

4. Neuhuber W, Mclachlan E, Jänig W (2017) The sacral autonomic outflow is spinal, but not "sympathetic". Anat Rec 300(8):1369-1370

5. Jänig W, Keast JR, McLachlan EM, Neuhuber WL, SouthardSmith M (2017) Renaming all spinal autonomic outflows as sympathetic is a mistake. Auton Neurosci 206:60-62

6. Espinosa-Medina I, Saha O, Boismoreau F, Brunet J-F (2017) The "sacral parasympathetic": ontogeny and anatomy of a myth. Clin Auton Res https://doi.org/10.1007/s10286-017-0478-7 